\title{
Author's Index
}

(Numbers refer to abstract numbers)

Abascal J. 93 Adamicza A. 49 Adamsons R. J. 8 Alexandre G. P. J. 24 Anderson C.B. 23

Antonini L. 30 Appel G. 80 Arfors K. E. 47 Arif S. 8 Asp N.G. 45 Aune S. 64

Babitch A. 8 Baer U. 69 Basuet J. 74 Basuet J. 68 Baird R.N. 18 Balabaud C. 27 Balabaud C. 1 Baron D.P. 83 Barreiro J. J. 118 Barzilai A. 15 Bateson P.G. 95 Bauer H. G. 45 Bauknecht K.J. 69 Bauminger B. 18 Beaubernard C. 5 Beck Ch. 86 Beckenlechner P. 113 Geger H.G. 10 Belenger J. Ill Belleville J. 68, 74 Belliard R. 1 Bellusci R. 30 Benichoux R. 131 Benoist F. Ill Beretta E. 63 Berg M. 92 Bernardi M. 30 Bernhardt-Huth D. 25 Bernhard A. 86 Bertrand Y. 88 Beyer J. 113

Biesmans M. 58 Bijnen A.B. 78 Binet J.P. 121 Bioulac P. 1 Birk M. 141 Birk M. 117 Bismuth H. 5,101 Bittner R. 10 Blake G. 65 Block G.E. 41 Blumel G. 117, 125, 141 Boccalon H. 17 Bockhorn H. 83 Bohler N. 123 Bohuon C. 5 Bolewski J. 99 Bore P.J. 22 Borgers M. 116 Boschat M. 7 Boucays A. 17 Bouma W. H. 133 Bracq H. 122 Bradley D. M. 12 Brandt M. 50 Braun E. 110 Brendel W. 102 Brenken U. 53 Breu E. 141 Bruch H. P. 36, 40 Bruckner U. B. 50 Buchanan K. D. 95 Burk R. 60 Burri C. 124 Bursch J. 86 Bustamente I. 67 Butt K. N. H. 8 Buurman W.A. 79 Buzby G.P. 143

Carlet J. 96 Castillo-Olivares J.L.

100, 108, 118 Catier P. 122

Cavallari A. 30 Cerny J. 112 Chan L. 22 Chignier E. 20, 68 Cienfuegos J. A. 118 Cividini F. 145 Claes L. 124 Clark J. 134 Comtat M. 17 Conzen P. 113 Cooper A.J. 142 Cornillon B. 74 Couhig E. 82 Crocket A. 9 Cuschieri A. 134

Daenen W. 116 Dahlqvist A. 45 Dandona P. 54 Darragon T. 96 Davidson H. A. Debry G. 89 Decloitre F. 101 de Hemptinne B. 3 Deiber J. 89 Delin N.A. 6 Demierre D. 73 Dennis C. 8 Depaepe J. 120 Dessapt B. 68 Deuvaert F.E. 120 Di Padova C. 63 DiMagno E. P. 59 Dielen D. 58 Dioguardi M.L. 63 Dioguardi V. 30 Dionigi R. 145 Dociu N. 39 Dodi G. 57,91 Dominioni L. 145 Donaldson L. A. 62 Dostal M. 112 Doyle D. 9

Author's Index

Dozois R. R. 59 Drouin P. 89 Duran C.M.G. 109 Dureau G. 74, 119 Durliat H. 17

Eastcott H.H.G. 56 Ebata H. 126 Eloy R. 20, 68, 119 Endrich B. 137 Engeset A. 105 Enjalbert A. 17 Erhardt W. 125 Ericcson M. 16, 92 Esato K. 32 Etheredge E. E. 23

Fagniez P. L. 96 Falk A. 48 Farndon J. R. 93 Faugon H. 1 Fawwaz R. 84 Figuera D. 108, 118 Fischer J.E. 31 Fischer J.H. 21-26 Flameng 116 Fleitas M. 109 Foster K.J. 142 Franco D. 101 Fredlund P. E. 45 Fritsche H. M. 125 Fromm D. 13 Fruhling J. 132 Fuhs M. 21-26 Fukushima H. 130 Funk W. 113

Galkowska H. 103 Garcia-Sola R. 100 Gebhardt K. 110, 114 Geier G. 135 Gianello P. 24 Gigou M. 101 Giordan J. 90 Glattli S. 76 Go V.L.W. 59

Goh H. 97 Goodwin C.W. 138 Gotz A. 137 Graffner H. 11 Greep J. M. 79 Gregor Z. 112 Grenier J. F. 94 Gross J.F. 136 Groussard M. 7 Gruber U. F. 76 Gruca Z. 33 Grundschober F. 123 Guba P. 112 Guidollet J. 20 Gundermann K.J. 25, 28 Gundlach J. 4 Gut J.P. 27

Hagenfeldt L. 6 Hagl S. 110-114 Haglund U. 48 Haiges M. 67 Hamilton G. 54 Hammer C. 102 Hanson P. 132 Hantog Z. 49 Hanzelka P. 112 Hardy M. A. 80-84 Haring R. 4-69 Hartmannova 
B. 112 Hau T. 98 Hausen M. 115 Hedin H. 75 Heil K. 140 Heimisch W. 110-114 Heitland W. 67 Helbing G. 124 Hellema H.W.J. 37 Herfarth Ch. 60-135 Hernandez-Salvador C.

100 Heymer B. 60 Heynen Y.G. 119 Hildebrandt A. G. 4 Hirner A. 4

Hobbs K. E. F. 54 Hofbauer F. 14 Hoffman R. 98 Hoitsma H.F.W. 66 Homan van der Heide

J.N. 53 Horn J. 60 Huguet C. 7 Husson J.L. 122 Hutchinson F. 134 Hutton R. 54

Ingemansson S. 16-92 Intaglietta M. 136 Isselhard W. 21-26

Jakesz R. 14 James J. H. 31 Jarhult J. 16 Jeekel J. 79 Jensen U. 140 Jeppsson B. 31 Joffe S.N. 962 Johnston I.D.A. 93 Joling P. 78

Kaene F. B. 59 Kamiyama Y. 34 Kammerling R. M. 142 Karacsony G. 49 Karavias Th. 4 Karliczek G. F. 53 Karran S. 97, 142 Kawahara T. 107 Keaveny T.V. 19 Kern E. 40 Kim Y.Z. 25 Kimura K. 28 Kinnunen P.K.J. 61 Kirn A. 27 Kivilaakso E. 13, 61 Klebniczki J. 49 Klooker P. 115 Knight C.J. Ill Kobayasshi O. 32 Kohler U. 115

Author's Index

Kohno Y. 34, 139 Kolstad P. 105 Konertz W. 86 Konradt J. 69 Kort W.J. 81 Kradolfer S. 76 Kranendonk S. E. 42 Kujath P. 36 Kummer D. 67 Kusano M. 126

Lambert A. 88 Lambotte L. 88, 128 Lamesch A. J. 39 Lapinski J. 99 Larsbraaten M. 144 Laszcower M. 73 Laubenthal H. 75 Lauder I. 93 Lazowski J. 99 Le Clenent P. 88 Le Clerc J. L. 120 Le Gall J. R. 96 Lejeune F. J. 132 Lempinen M. 61 Lennquist S. 92 Lie T.S. 25, 28 Liedberg G. 11 Linacero G. 100 Lindahl J. 6 Linkola J. 129 Lise M. 57-91 Louisot P. 20 Lovig T. 144 Lukomska B. 105 Lundberg C. 47 Lusby R.J. 18 Lyndon P.J. 43 Lypka A. 99

Mahenc J. 17 Maier W. D. 50 Malt R. A. 44 Marchal G. 90 Marescaux J. 94 Martinez R. 109 Maruyama Y.29 Mathieu M. 132 Mathisen O. 64 Matsumoto H. 29 Maurer W. 115 Maza.Inza J.M. 46 Mazzioti A. 30 McKelvey S. T. D. 65 Meijer S. 66 Meisner H. 114 Mendler N. 110-114 Menon C. 57 Messmer K. 75-113-137 Meves M. 10 Militello C. 57-91 Miller E. 143 Minkowitz S. 8 Minton J.P. 104 Mirouze J. 90 Mito M. 126 Mittmann U. 50-115 Miyashita H. 32 Miyata M. 21-26 Mo A. 64 Mohri H. 32 Montero C.G. 108-118 Monies J.A. 118 Moore L. 97 Mora N.P. 108-118 Morales M. 109 Moreels R. 7 Morin J. 101 Morita N. 32 Morrison A. R. 23 Mud H.J. 42 Mulder J.H. 133 Mullen J.L. 143 Myrvold H. E. 48

Nagy S. 49 Nakache J. P. 96 Nakatani T. 34 Neugebauer R. 124 Ngala Kenda J.F. 128 Niessen G.J.C.M. 78 Nieuwenhuis P. 87

Niinikoski J. 70 Noordhoek J. 2 Norberg K.A. 52 Nordlinger B. 7 Nowygrod R. 80 Nylander G. 52

Obertop H. 42-78 O’Connor L.F. 108 Oettinger W. 140 Olek K. 28 Olszewski W.L. 51, 85, 103, 105 Oluwole S. 84 Onishi T. 126 Onursal E. 106 Orlowski T. 99 Oetega-Gayton M. 8 Osborn K. 77 Oscarson J. 11 Oste R. 45 Ozawa K. 34-139

Paillot J. 131 Paloheimo M.P.J. 129 Papa V. 30 Papacrhistodoulou A. J.

97 Papatheofanis I.D. 72 Parbhoo S. P. 127 Pardy B. J. 56 Parks T. G. 95 Parquet M. 7 Paul J. 74 Pecile A. 63 Pedrazzoli S. 57-91 Perissat J. 1-27 Pessina F. 57 Peters W. 136 Peter K. 75

Peterson-Dahl E. 44 Petrowicz O. 117 Pfeifer H.G. 141 Pfeiffer U. 117-141 Pfluger G. 123

Pham T. C. 90

Author's Index

Pilgreen K. 77 Pirson Y. 24 Planche Cl. 106-121 Plenk H. 123 Pointel J. P. 89 Pollet J. E. 35 Pomar J.L. 109 Powanda M.C. 138 Preece P.E. 134 Primo G. 120 Prokscha G.W. 125 Prop Jm. 87 Pruitt B.A. 138 Psaila J.V. 12 Puel P. 17

Radda G. K. 22 Raeder M. 64 Raekallio J. 71 Rasche A. 25 Raul F. 94 Rauwerda J. 66 Reemtsma K. 84 Renvall S. 70-71 Reynaert M. 88 Richter W. 75 Risberg Bo 77 Rodriguez Romen W. 36^0 Roots I. 4 Ross J. S. 44 Ross B.D. 22 Rousselle D. 89 Rozsa I. 54 Ruka M.P. 51 Rumke P. 37 
Salaman J. R. 82 Salomon F. 5 Salvador C.H. 108 Saric J. 1-27 Savunen T. 71 Schider S. 123 Schiessel R. 14-15 Schlag P. 135 Schmidt E. $36^{\wedge} 10$

Schoenberg M. H. 47 Schraut W.H. 41 Schrempf-Decker G.E.

83 Schroder T. 61 Sebening F. 110 Sehr P. A. 22 Selam J.L. 90 Sells R. A. 72 Semik M. 86

Senekowitsch A. 125 Senftleber I. 102 Sery G. 89 Sharp P. F. 35 Sheridan W. 19 Shimahara Y. 139 Sicard G. A. 23 Silen W. 13-15 Simmons R. L. 98 Skidmore R. 18 Sledzinski Z. 33

Smedegard G. 47 Sotolova O. 112 Sperti C. 91 Squifflet J. P. 24 Stalpaert G. 116 Starlinger M.

14 Steinberg J.J. 143 Stein T.P. 143 stemberger A. 125 Stepkowski S. 85 Stock C. 94 Stubinger B. 125 Sugitachi A. 107 Sundler F. 92 Suy R. 116

Tacconi C. 30 Tagaki K. 107 Takahama T. 29 Takeda J. 130 Takeda H. 34 Takeoka A. 130

Tanaka M. 107 Teixeira M. 136 Ten Kate F.S.W. 2

Terpstra O. T. $2^{\wedge} 14$ Thaw H.H. 47 Thulborn K. 22 Tobe T. 34-139 Trunet P. 96 Tudway A.J.C. 43 Turina M. 73

Ueberschlag P. 76 Uggeri F. 63 Uhlhaas S. 28 Ukikusa M. 34-139 Urbanek P. 112

Van Andel N.V. 38 Van den Broeke J.J. 53 Van den Dungen J.J.A.N. 53-55 Van der Linden C.S. 79 Van Cangh P. 24 Van Ermen H. 116 Van Hee R. 58 Van Houten H. 42 Vara-Thorbeck R. 46 Vasku J. 112 Vasseur M. 94 Vegt P. A. 79 Velders A.J. 55 Verhest A. 132 Verley J.M. 106 Vernhes G. 89 Veser J. 135 Vetter H. 115 Victor H. 50 Viljanto J. 71 Violet F. 20 Visser J.J. 66 Vitolo A. 30

Wada T. 29 Walter G. 140 Walters G. 18 Walther B. 11 Ware J. 52 Wax S.D. 77 Webb W. R. 77 Author's Index

Webster D.J.T. 104 Weijma I.M. 81 Weiss M. 106-121 Wellens F. 120 Wembacher J. 102 Westbroek D.L. 42-81 Westerhof N. J. W. 55 Wheeler M.H. 12 Wildevuur C.R.H. 38, 53, 55, 87 Williamson R.C.N. 43,

44 Wilmore D.W. 138 Wilson J.H.P. 2 Wisbey M. 134 Wood R.A.B. 134 Woodcock J. 18 Woods R.J. 127 Wriedt-Lubbe I. 125

Yamana H. 130 Yamato T. 139 Yatuka K. 130

Ziparo V. 31 Zoli G. 30 Zumtobel V. 140 Zychlinski L. 33 
B2264 Printed in Belgium by Ceuterick s.a.

Brusselse straat 153 B 3000-Louvain

A. Struyf Oude Baan 353 B 3000-Leuven 